\title{
How to Provide Adequate UV Dosimetry to Avoid Vitamin D Deficiency and Related Neuropsychiatric Disorders
}

\author{
Irina Terenetskaya* \\ Institute of Physics National Academy of Sciences of Ukraine, Ukraine
}

*Corresponding author: Irina Terenetskaya, Institute of Physics NAS Ukraine, 03028, Prospekt Nauki, 46; Kyiv, Ukraine.

Received Date: December 12, 2019

Published Date: December 17, 2019

\section{Abstract}

The essential role of vitamin D3 in maintaining calcium metabolism and other physiological processes in the body (modulation cell growth, neuromuscular conduction, immunity and inflammation) is well established. In recent studies vitamin D3 is considered as one of the neurosteroids that are required for the brain to develop and function properly. Vitamin D3 is synthesized in human skin from 7-Dehydrocholesterol (provitamin D3) upon solar UVB (280-315 nm) irradiation which is very variable depending on latitude, season, air pollution, etc. A high incidence of vitamin D deficiency in almost all populations in the world was detected in the last decade by epidemiologic studies that may cause a number of chronic diseases (cancer, multiple sclerosis, etc.). This indicates the need to measure precisely the vitamin D synthesizing capacity of sunlight (as well as artificial UV sources) to maintain optimal vitamin D status. For this purpose, a bioequivalent UV 'VitaD'-biodosimeter has been developed which is based on the same photoreaction in vitro by which vitamin D3 is synthesized in human skin.

Keywords: UV radiation; UV bioeffects; Erythema; Provitamin D photoconversion; Vitamin D deficiency; UV biodosimeter

Abbreviations: UV: Ultraviolet; 7-DHC - 7: Dehydrocholesterol Provitamin D3; 1,25(OH)2D: 1,25- Dihydroxyvitamin D (calcitriol); 25(OH)D: 25Hydroxyvitamin D (calcidiol); IU: International Unit for Vitamin D3 (1 IU = $40 \mathrm{mcg}$ ); CIE: International Commission on Illumination (Commission Internationale de L'Eclairage); MED: Minimal Erythemal Dose; LC: Liquid Crystal; SED: Standard Erythemal Dose

\section{Introduction}

Vitamin $\mathrm{D}$ belongs to the group of fat-soluble vitamins. It is naturally present only in a very limited amount of food, and its synthesis in the human body is possible only under certain conditions, when ultraviolet rays of sunlight enter the skin. Also known as calciferol, vitamin D appears in two major forms. Vitamin D2 (or ergocalciferol) is synthesized in plants, and Vitamin D3 (cholecalciferol) is synthesized in mammal skin. The terminology 'vitamin D' is employed here in a general sense. It is important to note that the photoinduced synthesis of these two types of steroids occurs by ideal analogy.

Vitamin D formed during exposure to the sunlight (and also obtained from food and in the form of food additives) is biologically inert. In order to activate and convert Vitamin D3 into the active form, two hydroxylation processes must take place in the body. At first Vitamin D3 is metabolized in the liver to 25-hydroxyvitamin
D (25(OH)D), which is a marker of vitamin D nutritional status (its optimal concentration should be $30-60 \mathrm{ng} / \mathrm{ml}$ ). Further hydroxylation occurs in the kidney and forms the biologically active 1,25-dihydroxyvitamin D (1,25(OH)2D) which is not a vitamin but is a steroid hormone that produces biological responses via binding to its receptor (VDR) in at least 37 tissues [1].

Many cells have vitamin D receptors, and in some tissues have their own $1 \alpha$-hydroxylase to form active forms of D-hormone and they can locally generate high intracellular concentrations of 1,25 $(\mathrm{OH}) 2 \mathrm{D}$ for their own purposes functioning without increasing its concentration in the general bloodstream. Recent studies show that vitamin $\mathrm{D}$ and $1 \alpha$-hydroxylase receptors are widespread in all parts of the brain, affecting the cognitive functions of the hypocampus [2]. In particular, there is enough evidence that falls in older people are associated with vitamin D deficiency (the concentration of $25(\mathrm{OH}) \mathrm{D}$ is below $10 \mathrm{ng} / \mathrm{ml}$ ). 
Selective sensitivity of vitamin D synthesis to the UVB part of solar spectrum makes almost impossible to give recommendations for effective solar exposure times because of daily, seasonal and latitudinal variability in the UVB intensity caused by changes in clouds, aerosols and air pollutions as well as personal sensitivity [3-4]. Insufficiency in solar UVB irradiation can lead to vitamin D deficiency in humans and thus create or increase existing health problems. Since the biological effectiveness of UV radiation depends significantly on the radiation wavelength (increasing with increasing energy of UV photons), the measurement of its biological effectiveness requires special tool whose spectral sensitivity is correlated with the action spectrum of a particular biological effect. This purpose is served by special biodosimeters which measure integrated biological effect directly, but the result is expressed in specific biological units.

Naturally, to prevent possible negative effects most attention is paid to the measurements of erythemic and mutagenic (carcinogenic) biological activity of UV radiation. The estimation of harmful UV levels is traditionally carried out by a variety of commonly used broadband UV detectors which have an output in terms of sunburn units (MEDs). Due to significant difference between the CIE erythema and 'Vitamin D synthesis' action spectra UV dosimetry using measured erythemic UV dose will not provide a satisfactory quantification of vitamin D synthesis especially considering complexity of the processes involved in vitamin D synthesis [4-6].

For this purpose original UV biodosimeter for an in situ monitoring of the vitamin-D-synthetic capacity of sunlight and/or artificial UV sources has been developed based on the same photoreaction in vitro by which vitamin $D$ is synthesized in human skin [7]. The next section will describe briefly the process of vitamin D synthesis and the UV D-biodosimeter $[8,9]$.

\section{Discussion}

Biological effect, i.e. the production of vitamin D is a two-stage process. The first step is photoinduced formation of previtamin D from provitamin D as a result of UV photons absorption. The second step is thermo-induced conversion of previtamin D into vitamin D. It is reasonable to assume that the amount of previtamin D accumulated during exposure to ultraviolet light can serve as a measure of the biologically effective ("anti-rachitic") dose of ultraviolet radiation.

But the task of determining the concentration of formed previtamin D is complicated by the fact that previtamin D itself is not stable to UV radiation and undergoes a number of side photoconversions. As a result, UV irradiation of initial provitamin $\mathrm{D}$ gives rise to the formation of a multicomponent photoisomer mixture. It is necessary to emphasize important features of the photoreaction [10]: (1) The accumulation of previtamin is nonlinearly dependent on a UV dose, (2) the maximum achievable concentration of previtamin $\mathrm{D}$ is highly dependent on irradiation wavelength (for monochromatic irradiation) or on the spectral composition of the UV lamp radiation. It is unlikely that any UV detector (an optoelectronic device or a photosensitive polymer film) can display correctly the above features and adequately measure the vitamin-D-synthetic activity even if their spectral sensitivity corresponds to the action spectrum of vitamin D synthesis.

Therefore, in our UV biodosimeter the 7-dehydrocholesterol molecules being targets for UV photons are embedded in specially designed UV transparent stable matrix used as photosensitive element; the dosimeter response to UV radiation is photoinduced conversion of provitamin D into previtamin D which is immediate precursor of vitamin D. As mentioned above, the biological "antirachitic" UV dose is determined by the amount of accumulated previtamin D. To follow the photoreaction course in real time three operation modes of varying complexity have been developed [11]:

- Mode 1: Direct measurement of Previtamin D concentration using original spectrophotometric analysis $[12,13]$

- Mode 2: Measurement of Previtamin D concentration using calibration graph $[14,15]$

- Mode 3: Visual detection of provitamin D photoconversion using LC matrix doped with 7-DHC [16,17]

To provide a missing link between in vivo and in vitro measurements direct measurements were performed of the vitamin D level in blood of healthy volunteers exposed to artificial UV source, in parallel with measurements of vitamin D synthesis in vitro. It is important to note that previtamin D synthesis in vitro was linked to in vivo 25(OH)D changes in human blood after sunbed exposure, and the linear correlation between the in vivo and in vitro data has been revealed [18]. Note also that a number of ultraviolet lamps which are widely used in phototherapy were tested on the 'antirachitic' activity along with the spectroradiometric measurements [19].

\section{Conclusion}

Hypovitaminosis D is associated with several neuropsychiatric disorders [20], and safe improvement of vitamin D status is especially important. The ability of 'Vitamin D' biodosimeter to provide reliable measurement of the 'antirachitic' solar and artificial UV radiation proves its applicability in a wide range of studies related to the biological effects of UV radiation and the vitamin D synthesis. As individual UV biodosimeter it might be helpful in epidemiologic and field studies for better understanding of the risks and benefits of solar exposure, as well as for comparison the effectiveness of oral vitamin D3 supplementation and UV exposure to find correspondence between units IU and SED.

\section{Acknowledgements}

I am grateful to all the co-authors of the publications listed below. Development of the UV Vitamin D-biodosimeter was supported by the EU (Grant No. IC20-CT96-0026) and by the Science and Technology Center in Ukraine (Projects Gr-50 and P344).

\section{Conflict of Interest}

There is no conflict of interest. 


\section{References}

1. Norman AW, Bouillon R (2010) Vitamin D nutritional policy needs a vision for the future. Exp Biol Med 235: 1034-1045.

2. Bischoff-Ferrari HA, P Burckhardt, K Quack-Loetscher, B Gerber, D I'Allemand, et al. (2012) Vitamin D deficiency: Evidence, safety, and recommendations for the Swiss population. Report written by a group of experts on behalf of the Federal Commission for Nutrition (FCN) 2012.

3. AR Webb, LW Kline, MF Holick (1988) Influence of season and latitude on the cutaneous synthesis of vitamin D3: exposure to winter sunlight in Boston and Edmonton will not promote vitamin D3 in human skin. J Clin Endocrinol Metabol 67: 373-378.

4. I Terenetskaya, T Orlova (2011) Variability of solar UV-B irradiance: in situ monitoring and model calculation of the vitamin D synthetic capacity of sunlight. Int J Remote Sens 32: 6205-6218.

5. M Norval, LO Bjorn, FR de Gruijl (2010) Is the action spectrum for the UV-induced production of previtamin D3 in human skin correct? Photochem Photobiol Sci 9: 11-17.

6. Terenetskaya (2003) Duality of solar UV-B radiation and relevant dosimetry: vitamin D synthesis versus skin erythema. Proc. SPIE 4896:144-150

7. Terenetskaya (1994) Provitamin D photoisomerization as possible UVB monitor: kinetic study using tunable dye-laser, Proc SPIE 2134B: 135140 .

8. ON Galkin, IP Terenetskay (1999) Vitamin D biodosimeter: basic characteristics and potential applications. J Photochem Photobiol B 53: 12-19.

9. IP Terenetskaya (2000) Spectral monitoring of biologically active solar UVB radiation using an in vitro model of vitamin D synthesis. Talanta 53: 195-203.

10. Jacobs HJC, Havinga E (1979) Photochemistry of Vitamin D and Its Isomers and of Simple Trienes. Adv Photochem 11: 305-373.
11. I Terenetskaya (2016) Three operation modes of the 'Vitamin-Dbiodosimeter' Proc. of SPIE, Vol. 9887 Biophotonics: Photonic Solutions for Better Health Care V. In: Jürgen Popp, Valery V Tuchin, Dennis L Matthews (Eds.), Francesco Saverio Pavone.

12. IP Terenetskaya, KO Terenetsky (1994) The method of bioactive ultraviolet radiation monitoring" Ukrainian Patent No. 94043235. B4401247

13. IP Terenetskaya, YuM Samchenko, TN Orlova, PS Kapinos, IE Boldeskul, et al. (2013) Method of determination of biologically active UV dose, Ukrainian Patent, Patent of Ukraine, p. 84586,

14. Terenetska IP, Orlova TM, Kirilenko EK, Galich GA, Eremenko AM (2011) Method and device for in situ determination of a vitamin-D-synthesizing amount of natural and artificial UV irradiation, Ukrainian Patent, p. 93569.

15. Terenetska IP, Orlova TM, Kirilenko EK, Galich GA, Eremenko AM (2013) A Method for In Situ Determination of the Vitamin-D-Synthetic Dose of Natural and Artificial Ultraviolet Irradiation and its implementation in a Personal Bio-dosimeter' Patent US 8,552,391 B2.

16. Terenetskaya (2006) Method of UV dosimetry and device for visual detection of bioactive UVB radiation, Ukrainian Patent № u 200600162.

17. IP Terenetskaya, MV Vasnetsov, PS Kapinos, DS Kasyanyuk (2017) 'Method of visual determination of 'antirachitic' biologic dose of ultraviolet radiation', Patent of Ukraine.

18. T Orlova, J Moan, Z Lagunova, L Aksnes, I Terenetskaya, et al. (2013) "Increase in serum 25-hydroxyvitamin-D3 in humans after sunbed exposures compared to previtamin D3 synthesis in vitro, J Photochem Photobiol B: Biology 122: 32-36.

19. Terenetskaya (2018) How to measure the Vitamin-D-synthetic activity of UV lamps used in phototherapy? Integr Mol Med 5(2): 1-5.

20. Harms LR, Burne THJ, Eyles DW, McGrath JJ (2011) Vitamin D and the brain. Best practice \& research. Clinical endocrinology \& metabolism 25(4): 657-669. 